\title{
Simulations of long time scale dynamics using the dimer method
}

\author{
Graeme Henkelman ${ }^{\mathrm{a}}$ and Hannes Jónsson ${ }^{\mathrm{a}, \mathrm{b}}$ \\ a Department of Chemistry, Box 351700, University of Washington, Seattle, WA 98195-1700, USA \\ b Faculty of Science, VR-II, University of Iceland, 107 Reykjavík, Iceland
}

May 27, 2001

\begin{abstract}
We have carried out long time scale simulations where the "dimer method" [G. Henkelman and H. Jónsson, J. Chem. Phys. 111, 7010 (1999)] is used to find the mechanism and estimate the rate of transitions within harmonic transition state theory and time is evolved by using the kinetic Monte Carlo method. Unlike traditional applications of kinetic Monte Carlo, the atoms are not assigned to lattice sites and a list of all possible transitions does not need to be specified beforehand. Rather, the relevant transitions are found on the fly during the simulation. An application to the diffusion and island formation of $\mathrm{Al}$ adatoms on an $\mathrm{Al}(100)$ surface is presented.
\end{abstract}

\section{INTRODUCTION}

One of the greatest challenges in computational studies of atomic systems is the simulation of long time scale evolution. While it is relatively straightforward to iteratively solve Newton's equations, the time scale that can be simulated that way, even when the simplest interaction potentials are used, is only on the order of nanoseconds for a typical system size and a weeks worth of CPU time on a modern computer. In chemistry and materials processing, most interesting transitions are thermally activated and take place on the time scale of microseconds or even seconds. The disparity of time scales is huge and it is clearly necessary to develop different methods for simulating time evolution.

Fortunately, there is often a separation of time scales. Vibrational motion of atoms occurs on the short time scale of femtoseconds. For a typical chemical reaction or diffusion event, there are on the order of $10^{10}$ vibrations before there is a sufficiently large fluctuation of thermal energy in the right degree of freedom for a transition to take place. Instead of following each vibration and waiting for these rare events, one can use transition state theory (TST) $[1,2,3,4,5,6]$ to calculate the average amount of time necessary for the system to make a transition. In order to calculate a rate, a bottleneck through which the system must pass in order to make the transition must be identified. This is the so-called transition state. For solid state systems, it is often possible to assume that the system is harmonic near the energy minimum representing the initial state and near a saddle point on the energy surface in the transition state. In this case, there is a simple form of TST referred to as harmonic transition state theory (hTST), in which the rate of a transition, $k$, can be directly related to properties of the initial state energy minimum and the saddle point $[7,8]$,

$$
k^{\mathrm{hTST}}=\frac{\Pi_{i}^{3 N} \nu_{i}^{\text {init }}}{\Pi_{i}^{3 N-1} \nu_{i}^{\ddagger}} e^{-\left(E^{\ddagger}-E^{\mathrm{init}}\right) / k_{B} T} .
$$




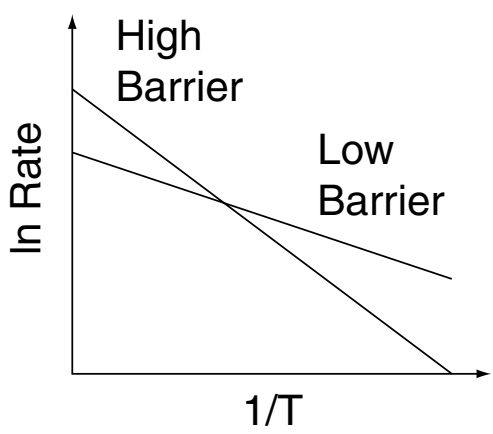

Figure 1: The temperature dependence of the rate of two different transitions is shown. The transition with the lower energy barrier corresponds to the line with shallower slope. A typical situation is shown in which the higher barrier transition has a higher prefactor (intercept with vertical axis). At low temperature, on the right side of the graph, the rate of the low barrier transition will be higher than that of the high barrier transition, and the dynamics will be dominated by events with low activation energy. At high temperature, the dynamics will be dominated by the higher entropy transition which has higher activation energy. This illustrates why a system cannot simply be heated to find events that occur on a longer time scale at low temperature.

Here, $E^{\ddagger}$ is the energy of the saddle point, $E^{\text {init }}$ is the local potential energy minimum corresponding to the initial state, and the $\nu_{i}$ are the corresponding normal mode frequencies. The symbol $\ddagger$ refers to the saddle point. All the quantities can be evaluated from the potential energy surface, at zero temperature, but thermal and entropic effects are included through the harmonic partition functions. The most challenging part in this calculation is the search for the relevant saddle point(s).

One possible method of increasing the rate of rare events is to simply heat the system. If the atoms have more thermal energy, they will be more likely to undergo transitions. Unfortunately this technique can lead to qualitatively different dynamics than at the lower temperature of interest. The situation is illustrated in Fig. 1. The temperature dependence of the rate of two different transitions is shown, one with a low activation energy and the other with a high activation energy. At low temperature, the low barrier transition will have a higher rate and dominate the dynamics. At high temperature, entropy becomes more important and the transition with a larger prefactor dominates, namely the high barrier transition.

Several methods have been proposed for accelerating the dynamics of rare events. Voter has developed several important methods. One is hyperdynamics $[9,10]$ which involves the creation of a repulsive bias potential which fills in regions around potential energy minima but does not affect the potential energy rims separating the energy basins, including the saddle points. In another method, proposed by Sorensen and Voter, so-called temperature accelerated dynamics (TAD) [11], the temperature of the system is increased, but the dynamics are carried out in such a way that only the transitions that would have occurred at a lower temperature are extracted. Finally, Voter has developed a method called parallel replica dynamics in which many trajectories are run in parallel while waiting for transitions to occur [12]. This method accelerates the simulation time required to see a transition by using many processors.

In this work we combine the dimer method [13] for finding saddle points and rate calculations within hTST with a kinetic Monte Carlo (KMC) method for simulating the evolution of systems over long time scales. This method is easy to implement and, compared to existing methods, may require less computational time for small systems. 


\section{THE LONG TIME DYNAMICS METHOD}

In order to carry out the simulation of a transition in the system, the rate and mechanism of all the relevant transitions from a given initial state need to be known. Within hTST this corresponds to finding all the low energy saddle points on the rim of the potential energy basin corresponding to the initial state. The dimer method is used to search for these saddle points.

\subsection{THE DIMER METHOD}

In the long time scale calculations presented here, between 25 and 50 dimer searches were launched from each new potential minimum visited. Dimer searches were started from configurations close to potential minima. The easiest way to choose a starting position is to make a random displacement away from the minimum. If all the atoms in the system are included in the random displacement, the dimer search can be biased towards finding higher energy transitions which involve many atoms. We find that it is better to displace only atoms within a local region. For each dimer search in the $\mathrm{Al}(100)$ ripening calculations (described in section 4), an under coordinated surface atom was chosen at random to be the center of the initial, local displacement. The displacements had a Gaussian distribution with a mean of $0.2 \AA$ in each degree of freedom. The region consisted of the central atom and its first and second neighbors. We have found that a continuous distribution is better than a fixed displacement size because it increases the variety of saddle points found. In principle, a scheme like this can eventually find all saddle points around a minimum simply because the starting points of the dimer search can be at any point in space. In practice, there is no guarantee that a complete list of saddle points can be found in a reasonable amount of time.

The dimer method is described in more detail in a previous publication [13]. The dimer is made up of two images (replicas) of the system. These images are separated in space by a finite distance displacement along a vector $\hat{\mathbf{N}}$. For an empirical potential this can be small. Here we have used $0.005 \AA$. Initially $\hat{\mathbf{N}}$ has non-zero elements only in the degrees of freedom along which the system was displaced.

There are two parts to each dimer move. The first part is dimer rotation. The lowest energy orientation of the dimer is along the lowest curvature mode. If the dimer is free to rotate, the forces acting on the two images will pull the dimer to the lowest curvature mode. This is done by defining a rotational force which is the difference in the force at the two images. Minimizing the energy of the dimer with respect to this rotational force aligns the dimer with the lowest curvature mode (this feature was used by Voter in his construction of bias potentials in hyperdynamics [10]). A modified Newton's method can be used to make this rotation efficient [13]. An important aspect of the dimer method is that it only requires the first derivative of the energy, not the second derivatives.

The second part of the algorithm is translation of the dimer. A first order saddle point on a potential surface is at a maximum along the lowest curvature direction and a minimum in all other directions. In order to converge to a saddle point, the dimer is moved up on the potential energy surface along the lowest curvature mode, but down in all other directions. This is done by defining an effective force on the dimer in which the true force due to the potential acting at the center of the dimer has the component along the dimer inverted. Minimizing with respect to this effective force moves the dimer to a saddle point. With empirical potentials, minimization using the conjugate gradient method works well.

It is not necessary to fully converge the dimer orientation at each translational step. We have found it most efficient to pick a certain tolerance for the rotational force. This sets how precisely the dimer is oriented along the lowest curvature mode before it is moved. The dimer is rotated at least once and possibly a few times until the rotational force is less than the specified tolerance. In

AA8.1.3 
these calculations the maximum rotational force was set at $5 \mathrm{meV} / \AA$.

The dimer requires on average 400 force evaluations to converge on a saddle point. Other methods for finding saddle points using only first derivatives of the energy have been proposed $[14,15]$, but it is unclear how their efficiency compares with the dimer method.

\subsection{KINETIC MONTE CARLO}

KMC is a powerful method that can be used to extend the timescale of simulations far beyond the vibrational timescale. In a typical KMC simulation, all transitions that can ever occur in the system, along with their rates, must be known before the simulation starts. Then, given any configuration of the system, a table of all possible transitions and corresponding rates needs to be constructed. The rates can be estimated using TST if the energy surface is known [16]. The problem here is to identify and tabulate all relevant transitions ahead of time. This limits the applicability of the method to simple systems where it is possible to guess which transitions are important. Systems that can undergo complicated transitions involving several atoms, such as the aluminum system described in section 3 , or in which atoms do not sit at lattice sites are extremely difficult to model with this approach.

If a list of possible transitions for a given initial state is available, a random number can be used to choose one of the transitions and let the system evolve to a new state. The probability of choosing a transition is proportional to its rate, $r_{i}$. On average, the amount of time that would have elapsed in order for this transition to occur is

$$
\Delta t=\frac{1}{\sum r_{i}}
$$

which is independent of which transition got chosen. It may also be important to include the appropriate distribution of escape times. For random uncorrelated transitions, this is a Poisson distribution. If $\mu$ is a random number from 0 to 1 , the elapsed time for a particular transition is given by

$$
\Delta t=\frac{-\ln \mu}{\sum r_{i}}
$$

The system is then advanced to the final state of the chosen transition and the process then repeated.

\subsection{COMBINED DIMER AND KINETIC MONTE CARLO}

The dimer method can be used to relax some of the limitations of the traditional implementation of the KMC scheme. If the dimer method is used to find possible transitions, there is little limitation on the complexity in terms of the number of atoms or the spatial extent of the transition. Also, the energy barriers do not need to be known before the calculation is started. Furthermore, the atoms do not need to be mapped onto a lattice and it is not necessary to anticipate all possible states of the system.

When a new minimum structure is visited, a swarm of dimer searches is sent out. For the calculations described in section 4, either 25 or 50 dimer searches were used. The results of the searches are collected. The system is quenched on either side of each saddle point in order to verify that it lies on a minimum energy path from the given initial state minimum. In the same way as described in section 2.2, a transition is chosen from the list, the system is advanced to the final state of that transition, and the time interval associated with the transition is added to the accumulated time.

AA8.1.4 


\section{APPLICATION TO AL(100) DIFFUSION}

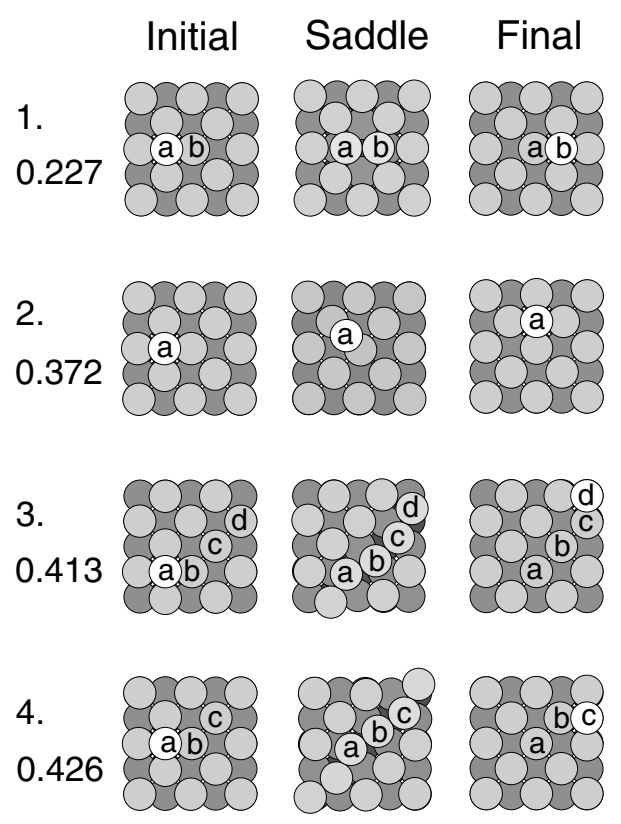

Figure 2: The four lowest energy transitions found with the dimer method for the diffusion of an $\mathrm{Al}$ adatom on $\mathrm{Al}(100)$. For each transition, the initial state, the saddle point configuration, and the final state are shown. Atoms are shaded by depth and the atoms with the greatest movement in each transition are labeled. The energy is given in $\mathrm{eV}$. The lowest energy diffusion process is a two-atom concerted displacement (1). The hop (2) is of similar energy to a concerted displacement involving thee (3) and four atoms (4). Because adatoms can so easily displace atoms in the surface, the $\mathrm{Al}(100)$ surface displays a great variety of transitions which would be hard to find by guesswork.

Diffusion and island formation of $\mathrm{Al}$ adatoms on an $\mathrm{Al}(100)$ surface was chosen as a test problem for this simulation method for a couple of reasons. First, a successful embedded atom potential of the Voter and Chen form exists for aluminum [17]. Also, this system has been extensively studied with the dimer method in reference [13]. There, it was shown that there are many transitions possible even for a single adatom on the $\mathrm{Al}(100)$ surface. The four lowest energy transitions found with the dimer method are shown in Fig. 2. A particularly interesting aspect of the $\mathrm{Al}(100)$ system is that an concerted displacement process has a lower energy barrier than the direct hop. This was shown by Feibelman with density functional theory calculations [18].

The dimer method was able to find a wide range of transitions from an initial state consisting of a single adatom in a four fold hollow site on $\mathrm{Al}(100)$. The substrate consists of 300 atoms, 50 per layer in 6 layers. The bottom two layers are held frozen and the top surface is left open to vacuum. Sixty different transitions were found with 1000 dimer searches. On average the low energy processes: the concerted displacement involving two, three and four atoms, and the hop, were found three quarters of the time. One quarter of the time a wide collection of higher barrier transitions were found.

In the KMC scheme, it is important to find all the relevant transitions, i.e. transitions with low activation energy. Using the statistics found for the single adatom diffusion events, there is an $80 \%$ certainty of finding all four (degenerate) two-atom concerted displacements with 50 dimer searches. For an accurate simulation, this indicates that there should be many dimer searches at 

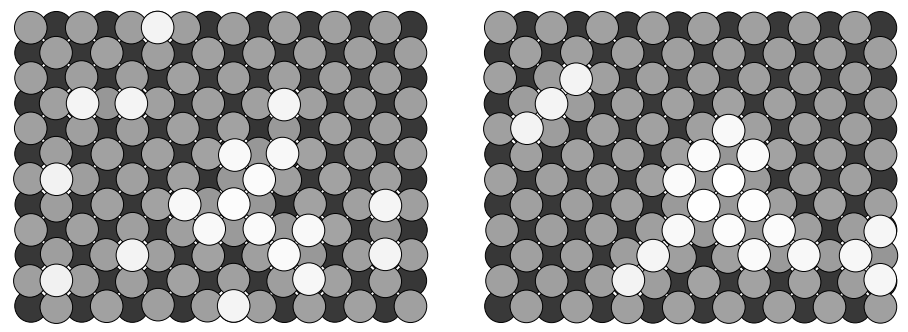

$$
\mathrm{n}=1 \quad \mathrm{t}=0 \mathrm{~ns}
$$

$$
\mathrm{n}=10 \quad \mathrm{t}=6 \mathrm{~ns}
$$
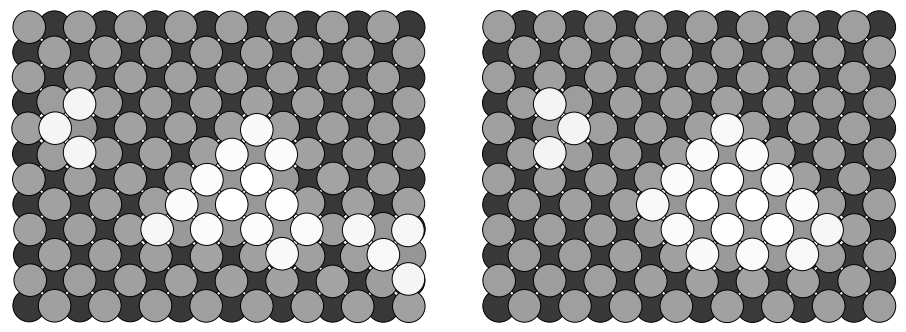

$$
n=344 \quad t=70 n s
$$

$$
\mathrm{n}=1000 \mathrm{t}=8 \mu \mathrm{s}
$$

Figure 3: Snapshots from a simulation of $\mathrm{Al}$ adatoms on an $\mathrm{Al}(100)$ surface. Initially twenty atoms were deposited at random on the surface. After $6 \mathrm{~ns}$ (10 transitions) all the adatoms have merged to form clusters. After $70 \mathrm{~ns}$ (344 transitions) a large, compact island has formed, but there are still two outlying islands. The trimer in the upper left has four possible rearrangements with a low activation energy. Many of the 344 transitions correspond to these rearrangements, but since old configurations are stored during the simulation the repeated transitions do not require new dimer searches. After $8 \mu \mathrm{s}$ (1000 transitions) the large island has taken a more compact shape and merged with one of the smaller islands. Of the 1000 total transitions in the simulation, 139 were distinct configurations and required new dimer searches.

each step. Fortunately, they can easily be carried out in parallel on separate computers. But, even with a modest number of searches, the method can give a good, qualitative idea of how the system will behave over long time intervals.

\section{ISLAND RIPENING}

The results of a kinetic Monte Carlo simulation coupled with dimer searches is shown in Fig 3. Initially 20 atoms were randomly deposited on the $\mathrm{Al}(100)$ surface using classical dynamics. Then the system was quenched to the local energy minimum. This configuration is shown in the first panel $(n=1)$. The time evolution of the system at $300 \mathrm{~K}$ was then simulated. For each transition, 25 dimer searches were used to find the possible transitions. On average, 17 distinct transitions were found from the 25 dimer searches. When 50 dimer searches were used, this number rose to 23, showing that some transitions were missed when only 25 searches were carried out. During the first 10 transitions, the adatoms move via the concerted displacements or hops to form clusters. After 344 transitions, a large island has formed, but two smaller islands also exist. After 1000 transitions, one of the small clusters has merged with the large island which has taken a more compact shape. It takes nearly 8000 transitions before the other three-atom island merges with the larger island.

Of the first 1000 transitions, only 139 brought the system to a new state. Many transitions 


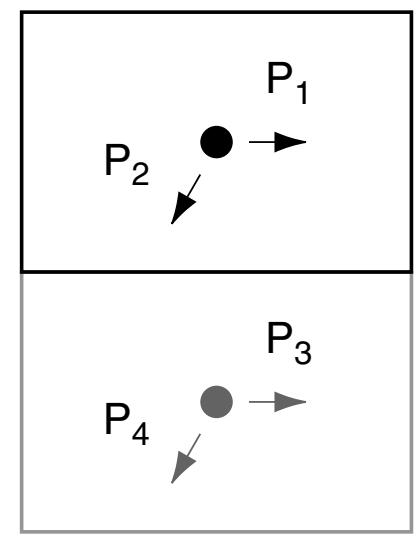

Figure 4: In a system of twice the size, there are on average twice as many available transitions. If $\mathrm{P}_{1}$ and $\mathrm{P}_{2}$ are possible transitions in the original system, there are two more transitions, $\mathrm{P}_{3}$ and $\mathrm{P}_{4}$, possible in the larger system. In the simplest possible implementation of the simulation method, this means that the number of dimer searches increases linearly with system size. But, if the transitions are local, only a limited region of the system is affected and will require new dimer searches. Also, saddle points closer to the transition region may only be slightly affected and previously found saddle point configurations can be reconverged with just a few dimer iterations. The number of dimer searches needed per transition does not, therefore, increase with system size. However, the time increment for each transition is only half as large in the larger system.

involved rearrangements of the three-atom cluster shown in the last two panels of Fig. 3. After each transition, the final state is compared to a table of all the previous configurations of the system. If the state has been seen before, no dimer searches are performed. Rather, the next transition is chosen from the old list of possible transitions. In this way, repeated transitions do not contribute significantly to the computational time, even if they occur frequently.

\section{EFFICIENCY}

It is important to know how a simulation method scales with system size. To determine this, we can consider how the number of force evaluations changes when the system is doubled in size. Force evaluations are considered rather than total computational time, because the evaluation of the force can scale differently depending upon the complexity of the interaction potential. In favorable situations, evaluation of the force scales linearly with system size.

Figure 4 shows an illustration of a system which has been doubled in size by joining two identical replicas of the system. In the larger system, there are twice as many possible transitions. In the crudest implementation of the simulation method, the number of dimer searches would then increase in proportion to the system size. If, however, the transitions are local, i.e. they only involve a small subset of the atoms, then large portions of the system will be unaffected by any one transition. Transitions that can take place in these unaffected regions will not require repeated dimer searches for saddle points. Rather, only the transitions which are affected by the last transition need to be updated and new transitions need to be added only for the region where the last transition took place. With the dimer method, it is also relatively straight forward to reconverge a set of known saddle points which have changed only slightly because of a nearby transition. Therefore, if the transitions are local, the number of new dimer searches that need to be carried out after a transition has occurred will not change as a function of the system size.

AA8.1.7 
Another aspect to the scaling is the time increment at each transition. When the system size is doubled, there are on average twice as many transitions possible. The time increment for each transition will then be half as long, as can be seen from Eq. 2. If the simulation is carried out in such a way as to cover a fixed time interval, then twice as many transitions need to be simulated in a system that is double in size. The number of force evaluations required to simulate a fixed length of time, therefore, scales linearly with system size.

This method for carrying out long time scale dynamics simulations is efficient enough for it to be implemented with first principles calculations of atomic iterations such as density functional theory. We have implemented the method in the VASP code $[19,20,21,22]$. More information about the implementation can be found on the web site http://ikazki01.chem.washington.edu/vasp/. We are currently applying the technique to the formation and break-up of boron clusters in silicon [23] and the diffusion of self-trapped excitons in silica glass [24].

\section{ACKNOWLEDGMENTS}

We would like to thank Art Voter, Normand Mousseau and Gerard Barkema for many useful discussions. This work was supported by NSF-KDI grant 9980125. 


\section{References}

[1] H. Eyring, J. Chem. Phys. 3, 107 (1935).

[2] E. Wigner, Trans. Faraday Soc. 34, 29 (1938).

[3] J. C. Keck, Adv. Chem. 13, 85 (1967).

[4] P. Pechukas, in Dynamics of Molecular Collisions, edited by W. Miller (Plenum Press, N.Y., 1976), part B.

[5] A. F. Voter and D. Doll, J. Chem. Phys. 80, 5832 (1984).

[6] A. F. Voter and D. Doll, J. Chem. Phys. 82, 80 (1985).

[7] C. Wert and C. Zener, Phys. Rev. 76, 1169 (1949).

[8] G. H. Vineyard, J. Phys. Chem. Solids 3, 145 (1957).

[9] A. F. Voter, J. Chem. Phys. 106, 4665 (1997).

[10] A. F. Voter, Phys. Rev. Lett. 78, 3908 (1997).

[11] M. R. Sørensen and A. F. Voter, J. Chem. Phys 112, 9599 (2000).

[12] A. F. Voter, Phys. Rev. B 57, R13985 (1998).

[13] G. Henkelman and H. Jónsson, J. Chem. Phys. 111, 7010 (1999).

[14] R. Malek and N. Mousseau, Phys. Rev. E 62, 7723 (2000).

[15] L. J. Munro and D. J. Wales, Phys. Rev. B 59, 3969 (1999).

[16] A. F. Voter, Phys. Rev. B 34, 6819 (1986).

[17] A. F. Voter and S. P. Chen, Mat. Res. Soc. Symp. Proc. 82, 2384 (1987).

[18] P. J. Feibelman, Phys. Rev. Lett. 65, 729 (1990).

[19] G. Kresse and J. Hafner, Phys. Rev. B 47, 558 (1993).

[20] G. Kresse and J. Hafner, Phys. Rev. B 49, 14251 (1994).

[21] G. Kresse and J. Furthmüller, Comput. Mater. Sci. 6, 16 (1996).

[22] G. Kresse and J. Furthmüller, Phys. Rev. B 54, 11169 (1996).

[23] G. Henkelman, B. P. Uberuaga, and H. Jónsson (unpublished).

[24] K. Tsemekhman, G. Henkelman, and H. Jónsson (unpublished). 